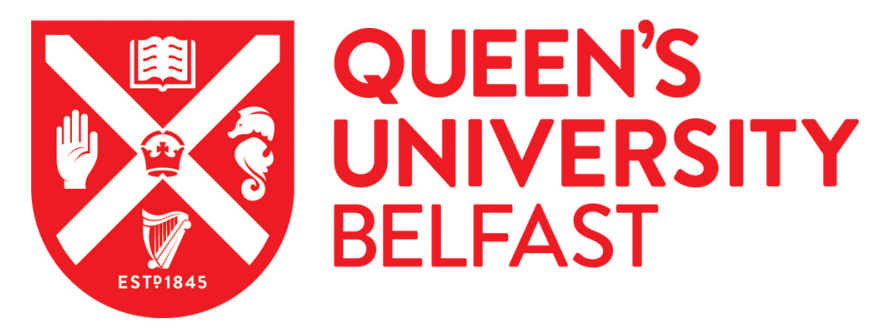

\title{
van der Waals coefficients for positronium interactions with atoms
}

Swann, A. R., Ludlow, J. A., \& Gribakin, G. F. (2015). van der Waals coefficients for positronium interactions with atoms. Physical Review A (Atomic, Molecular, and Optical Physics), 92(1), [012505].

https://doi.org/10.1103/PhysRevA.92.012505

Published in:

Physical Review A (Atomic, Molecular, and Optical Physics)

Document Version:

Peer reviewed version

Queen's University Belfast - Research Portal:

Link to publication record in Queen's University Belfast Research Portal

Publisher rights

(02015 American Physical Society

This work is made available online in accordance with the publisher's policies. Please refer to any applicable terms of use of the publisher.

\section{General rights}

Copyright for the publications made accessible via the Queen's University Belfast Research Portal is retained by the author(s) and / or other copyright owners and it is a condition of accessing these publications that users recognise and abide by the legal requirements associated with these rights.

Take down policy

The Research Portal is Queen's institutional repository that provides access to Queen's research output. Every effort has been made to ensure that content in the Research Portal does not infringe any person's rights, or applicable UK laws. If you discover content in the Research Portal that you believe breaches copyright or violates any law, please contact openaccess@qub.ac.uk. 


\title{
van der Waals coefficients for positronium interactions with atoms
}

\author{
A. R. Swann, ${ }^{*}$ J. A. Ludlow, ${ }^{\dagger}$ and G. F. Gribakin ${ }^{\ddagger}$ \\ Centre for Theoretical Atomic, Molecular, and Optical Physics, \\ School of Mathematics and Physics, Queen's University Belfast, Belfast BT7 1NN, United Kingdom
}

\begin{abstract}
The random-phase approximation with exchange (RPAE) is used with a $B$-spline basis to compute dynamic dipole polarizabilities of noble-gas atoms and several other closed-shell atoms (Be, $\mathrm{Mg}$, $\mathrm{Ca}, \mathrm{Zn}, \mathrm{Sr}, \mathrm{Cd}$ and $\mathrm{Ba}$ ). From these, values of the van der Waals $C_{6}$ constants for positronium interactions with these atoms are determined and compared with existing data. After correcting the RPAE polarizabilities to fit the most accurate static polarizability data, our best predictions of $C_{6}$ for Ps-noble-gas pairs are expected to be accurate to within $1 \%$, and to within a few per cent for the alkaline earths. We also used accurate dynamic dipole polarizabilities from the literature to compute the $C_{6}$ coefficients for the alkali-metal atoms. Implications of increased $C_{6}$ values for Ps scattering from more polarizable atoms are discussed.
\end{abstract}

PACS numbers: 36.10.Dr,34.20.Cf,34.50.-s

\section{INTRODUCTION}

The interaction of positronium (Ps) with matter and antimatter is an important topic [1] with applications in many areas of physics. For instance, the proposed AEgIS experiment would produce an antihydrogen beam from the reaction between Ps and antiprotons [2, 3]. The antihydrogen would then be used to determine whether antimatter is affected by gravity in the same manner as matter. Ps is widely used in condensed matter physics to determine pore sizes in microporous materials and probe intermolecular voids in polymers [4]. Further, positronium formation in porous materials is used to study its interactions with gases, e.g., xenon $[5,6]$, or the interaction between the Ps atoms themselves, with prospects of Bose-Einstein condensation at room temperature [7, 8].

Here we focus on the problem of Ps-atom scattering. Compared with electron-atom scattering and positronatom scattering, Ps-atom scattering is more difficult to treat theoretically, chiefly because both scattering objects have an internal structure [9].

In this paper we address low-energy Ps scattering from closed-shell atoms. The short-range Ps-atom interaction is repulsive, because (a) the Pauli principle prevents the electron from Ps from entering the volume occupied by the atomic electrons, and (b) the positron is repelled by the screened potential of the atomic nucleus. However, low-energy Ps-atom scattering is also affected by the attractive long-range van der Waals interaction [10-12].

The van der Waals potential behaves asymptotically as

$$
U(R) \simeq-\frac{C_{6}}{R^{6}}
$$

\footnotetext{
* Email: aswann02@qub.ac.uk

$\dagger$ Present address: AquaQ Analytics, Suite 5, Sturgeon Building, 9-15 Queen Street, Belfast BT7 1NN, United Kingdom

‡Email: g.gribakin@qub.ac.uk
}

where $R$ is the distance between the centers of mass of the atoms, and $C_{6}$ is the van der Waals coefficient for the atomic pair [13]. The value of the $C_{6}$ constant determines the scattering phase shifts for the partial waves $l \geq 2$ at low collision momenta $k$ [14],

$$
\delta_{l}(k) \simeq \frac{6 C_{6} \pi k^{4}}{(2 l-3)(2 l-1)(2 l+1)(2 l+3)(2 l+5)} .
$$

The magnitude of $C_{6}$ also affects the Ps-atom scattering length $A$. This can be seen from the estimate which uses the potential (1) with a cut-off at $R=R_{0}$ [12],

$$
A=\left(\frac{m C_{6}}{8}\right)^{1 / 4} \frac{\Gamma(3 / 4)}{\Gamma(5 / 4)} \frac{J_{-1 / 4}\left(x_{0}\right)}{J_{1 / 4}\left(x_{0}\right)},
$$

where $m$ is the reduced mass, $\Gamma$ and $J_{\nu}$ are the gamma and Bessel functions, respectively, and

$$
x_{0}=\frac{\sqrt{m C_{6} / 2}}{R_{0}^{2}} .
$$

The dimensional prefactor in Eq. (3) determines the characteristic magnitude of the scattering length in atomic collisions [15]. We use atomic units throughout, so that $m=2$ for Ps-atom collisions.

Mitroy and Bromley [16] calculated the $C_{6}$ constants for Ps-noble-gas interactions using the oscillator strength sum rule. For He they calculated the oscillator strengths via the configuration interaction (CI) method, while for $\mathrm{Ne}, \mathrm{Ar}, \mathrm{Kr}$, and Xe recourse was made to a set of published pseudo-excitation energies and dipole oscillator strengths [17], leading to semi-empirical (SE) values of $C_{6}$. In a recent paper devoted to Ps scattering from $\mathrm{Ar}$ and $\operatorname{Kr}[12]$, Fabrikant and Gribakin used the London formula [18] for the van der Waals coefficients, which gave values about $6 \%$ greater than those from Ref. [16]. To estimate the effect of this difference on Ps-atom scattering we can use Eq. (3), which shows that for $\operatorname{Ar}\left(R_{0}=2.67\right.$ a.u. [12]), changing $C_{6}$ from 152 a.u. [12] to 144.1 a.u. [16] increases the scattering length by about $4 \%$. This example shows that Ps-atom scattering is sensitive to the value of the van der Waals coefficient. 
In this work, we employ the random-phase approximation with exchange (RPAE) $[19,20]$ to calculate the dynamic dipole polarizabilities of the noble-gas atoms and several other closed-shell atoms with an $n s^{2}$ valence shell. These are used to calculate the $C_{6}$ constants for Ps-atom pairs ab initio. The RPAE method, often called simply the random-phase approximation (RPA), is equivalent to the (linearized) time-dependent coupled Hartree-Fock method (see, e.g., Ref. [21] and references therein). RPAE is known to provide a good description of atomic polarization for the noble gases $[22,23]$. To make our predictions more accurate, we use scaling based on the known values of the static dipole polarzabilities. As a result, we expect our final $C_{6}$ constants to be accurate to better than $1 \%$. As far as we are aware, there are currently no published values of $C_{6}$ for Ps interactions with other closed-shell atoms. Although the RPAE method is less accurate for them, our final $C_{6}$ values provide a good benchmark for future work.

\section{THEORY}

The van der Waals coefficient for a pair of atoms, $A$ and $B$, may be expressed as

$$
C_{6}=\frac{2}{3} \sum_{n, n^{\prime}} \frac{\left|\left\langle n_{A}|\mathbf{D}| 0_{A}\right\rangle\right|^{2}\left|\left\langle n_{B}^{\prime}|\mathbf{D}| 0_{B}\right\rangle\right|^{2}}{E_{n}^{A}+E_{n^{\prime}}^{B}-E_{0}^{A}-E_{0}^{B}}
$$

where $\mathbf{D}$ is the electric dipole operator, the matrix elements are taken between the ground $(0)$ and excited ( $n$ or $\left.n^{\prime}\right)$ states of the atoms, with respective energies $E_{0}$ and $E_{n}$, and the extra indices ( $A$ or $B$ ) are used to distinguish the two atoms [24].

There exists a useful relationship connecting dynamic polarizabilities of imaginary frequencies with $C_{6}$. The dynamic polarizability at frequency $\omega$ is given by

$\alpha(\omega)=\frac{1}{3} \sum_{n}\left(\frac{|\langle n|\mathbf{D}| 0\rangle|^{2}}{E_{n}-E_{0}-\omega-i \delta}+\frac{|\langle n|\mathbf{D}| 0\rangle|^{2}}{E_{n}-E_{0}+\omega+i \delta}\right)$,

where $\delta$ is a positive infinitesimal, which determines the sign of the imaginary part of $\alpha(\omega)$ for real frequencies $\omega$ above the ionization potential of the atom. The groundstate static polarizability is $\alpha(0)$. The van der Waals constant can be obtained by integrating the product of dynamic polarizabilities of atoms $A$ and $B$ over imaginary frequencies, viz.

$$
C_{6}=\frac{3}{\pi} \int_{0}^{\infty} \alpha_{A}(i \omega) \alpha_{B}(i \omega) d \omega
$$

This result is very convenient because the integration path avoids the poles of the dynamic polarizabilities.

For hydrogenic atoms (such as $\mathrm{H}$ and Ps), the polarizabilities are given by Eq. (6) with the dipole matrix elements replaced by the single-particle radial matrix elements $\langle n p|r| 1 s\rangle$, where $r$ is either the electron-proton
$(\mathrm{H})$ or electron-positron (Ps) separation. Thus, for an interacting pair of hydrogenic atoms, an essentially exact value of $C_{6}$ can be found. However, for many-electron atoms (such as the noble gases) the single-particle (e.g., Hartree-Fock) method does not give accurate results. RPAE is a many-body theory method that is known to give accurate dipole polarizabilities and photoionization cross sections for closed-shell atoms, with the best results for the noble gases [19, 22].

After the RPAE equations have been solved (see Appendix A for details), the dynamic dipole polarizability is calculated as

$$
\begin{aligned}
\alpha(\omega)=-\frac{2}{3}\left(\sum_{\nu>F, \mu \leq F} \frac{\langle\mu\|d\| \nu\rangle\langle\nu\|A(\omega)\| \mu\rangle}{\omega-\epsilon_{\nu}+\epsilon_{\mu}}\right. & \\
& \left.+\sum_{\nu \leq F, \mu>F} \frac{\langle\mu\|d\| \nu\rangle\langle\nu\|A(\omega)\| \mu\rangle}{-\omega+\epsilon_{\nu}-\epsilon_{\mu}}\right),
\end{aligned}
$$

where $\langle\nu\|d\| \mu\rangle$ is the reduced Hartree-Fock dipole matrix element, $\langle\nu\|A(\omega)\| \mu\rangle$ is the reduced RPAE dipole matrix element, $\epsilon_{\nu}$ is the energy of state $\nu$, and $i \delta$ has been dropped for $\omega=0$ and imaginary frequencies. In Eq. (8) the sums over the magnetic quantum numbers and spins have already been carried out, and the remaining sums are over the occupied $(\leq F)$ or empty $(>F)$ electron orbitals $\nu$ and $\mu$.

Note that the matrix element $\langle\nu\|A(\omega)\| \mu\rangle$ is calculated off-mass-shell, i.e., for $\omega \neq \epsilon_{\nu}-\epsilon_{\mu}$, and the energy differences in the denominator of Eq. (8) contain the unperturbed Hartree-Fock energies. An equivalent but more complicated approach would be to calculate these amplitudes on the mass-shell, simultaneously with finding the RPAE excitation energies $\omega_{\nu \mu}$. In this case the expression for the polarizability would contain modulus squared values of $\left\langle\nu\left\|A\left(\omega_{\nu \mu}\right)\right\| \mu\right\rangle$ and the RPAE excitation energies in the denominator [cf. Eq. (6)].

The expressions for $C_{6}$ and $\alpha(\omega)$ contain sums over the complete sets of excited states. In real systems, such as atoms, these sets of excited states include both discrete, Rydberg states and the continuum of states with energies above the ionization potential of the system. By using $B$ splines in a box of finite radius $R$ (see Section III), the continuum is discretized in a way that allows accurate numerical calculations of both the static polarizability (for $\omega=0$ ) and $\alpha(i \omega)$, and $C_{6}$.

\section{NUMERICAL RESULTS}

We use $B$-splines to construct either hydrogenic or Hartree-Fock basis states. They provide an accurate representation of the ground-state orbitals and an effective spanning of the continuum, due to an appropriately chosen radial grid (see, e.g., Ref. [25]). In this work we use a set of $60 B$-splines of order 9 , with a box size of $R=15$ a.u. The absence of true continuum states does not affect 
TABLE I. Static dipole polarizabilities $\alpha(0)$ for the noble gases and other closed-shell atoms (in atomic units).

\begin{tabular}{lrcccl}
\hline \hline Atom & Present & RPAE $^{\mathrm{a}}$ & RRPA $^{\mathrm{b}}$ & \multicolumn{1}{c}{ Rec. ${ }^{\mathrm{c}}$} & $F^{\mathrm{d}}$ \\
\hline $\mathrm{He}$ & 1.322 & 1.256 & 1.322 & 1.384 & 1.047 \\
$\mathrm{Ne}$ & 2.377 & 2.30 & 2.38 & 2.67 & 1.123 \\
$\mathrm{Ar}$ & 10.758 & 10.73 & 10.77 & 11.07 & 1.029 \\
$\mathrm{Kr}$ & 16.476 & 16.18 & 16.47 & 17.075 & 1.036 \\
$\mathrm{Xe}$ & 27.099 & 27.98 & 26.97 & 27.815 & 1.026 \\
\hline $\mathrm{Be}$ & 45.604 & 43.2 & 45.6 & 37.76 & 0.828 \\
$\mathrm{Mg}$ & 81.502 & - & 81.2 & 71.3 & 0.875 \\
$\mathrm{Ca}$ & 183.965 & 166 & 182.8 & 157.1 & 0.854 \\
$\mathrm{Zn}$ & 54.046 & - & 50.8 & 38.8 & 0.718 \\
$\mathrm{Sr}$ & 242.240 & - & 232.6 & 197.2 & 0.814 \\
$\mathrm{Cd}$ & 75.958 & - & 63.7 & 49.65 & 0.654 \\
$\mathrm{Ba}$ & 355.735 & - & 324.0 & 273.5 & 0.769 \\
\hline \hline
\end{tabular}

a This implementation $[22,26]$ suffers from poor convergence.

b Relativistic random-phase approximation [27].

c Recommended experimental values for the noble gases, $\mathrm{Zn}$, and Cd [28], and calculated values for alkaline earth metals [29].

${ }^{d} F$ is the ratio of the recommended value to the present value.

the accuracy of $\alpha(0)$ or $\alpha(i \omega)$ because all excitations in the sums are virtual (the denominator never vanishes); such virtually excited electrons cannot travel away from the atom, so describing them by a set of states in a box is accurate.

We calculated static dipole polarizabilities of $\mathrm{H}, \mathrm{Ps}$, the noble gases, and other closed-shell atoms and compared with exact theoretical or best experimental or theoretical values to verify the validity of the method. Then we computed dynamic polarizabilties $\alpha\left(i \omega_{j}\right)$ over a discrete set of imaginary frequencies

$$
\omega_{j}=\omega_{0}\left[e^{\sigma(j-1)}-1\right], \quad j=1, \ldots, N_{\omega},
$$

where $\omega_{0}, N_{\omega}$, and $\omega_{\max } \equiv \omega_{N_{\omega}}$ are parameters, and

$$
\sigma=\frac{1}{N_{\omega}-1} \ln \left(\frac{\omega_{\max }}{\omega_{0}}+1\right) .
$$

Values of $\omega_{0}=0.01$ a.u., $N_{\omega}=100$, and $\omega_{\max }=1000$ a.u. have been used throughout; these values were chosen to provide accurate, converged values of the integral (7). For $\mathrm{H}$ and $\mathrm{Ps}$ the polarizabilities were calculated essentially exactly; for the noble gases and $n s^{2}$ atoms they were calculated using the RPAE method. The values of $C_{6}$ were then found by evaluating Eq. (7) numerically.

The static polarizability obtained for $\mathrm{H}$ was $\alpha(0)=$ 4.500 a.u., in perfect agreement with the exact value of $\alpha(0)=9 / 2[24]$. The $B$-spline states and dipole amplitudes for hydrogen can be used to calculate $\alpha(0)$ and $\alpha(i \omega)$ for Ps by halving the energies and doubling the amplitudes (due to the reduced mass of Ps being a half of that of $\mathrm{H}$ ). This gives $\alpha(0)=36$ a.u. for Ps.

Table I shows the static polarizabilities obtained using RPAE for the noble-gas and other closed-shell atoms. The RPAE static polarizabilities for the noble-gas atoms agree to $0.1 \%$ or better with the results of the equivalent coupled Hartree-Fock calculation [30], with the
TABLE II. van der Waals $C_{6}$ coefficients for various Ps- $X$ systems (in atomic units).

\begin{tabular}{lrccc}
\hline \hline System & Present & Best prediction $^{\mathrm{a}}$ & CI/SE & Other $^{\mathrm{c}}$ \\
\hline Ps-H & 34.785 & - & 34.785 & 34.785 \\
Ps-Ps & 207.969 & - & - & 207.966 \\
\hline Ps-He & 12.849 & 13.41 & 13.37 & 14.6 \\
Ps-Ne & 23.759 & 26.48 & 26.74 & 27.4 \\
Ps-Ar & 96.212 & 98.69 & 98.50 & 104.4 \\
Ps-Kr & 142.185 & 146.71 & 144.1 & 155.1 \\
Ps-Xe & 222.355 & 227.38 & 221.6 & 240.6 \\
\hline Ps-Be & 241.416 & 208.6 & 210.7 & 294.3 \\
Ps-Mg & 393.484 & 355.8 & 357.9 & 506.4 \\
Ps-Ca & 717.138 & 641.5 & 636.6 & 1079 \\
Ps-Zn & 300.756 & 231.4 & - & 303.8 \\
Ps-Sr & 885.425 & 767.5 & 763.8 & 1144 \\
Ps-Cd & 397.161 & 285.0 & - & 381.6 \\
Ps-Ba & 1164.429 & 975.6 & 966.8 & 1569 \\
\hline \hline
\end{tabular}

a Obtained by scaling the matrix elements by $\xi$ and energies by $1 / \xi^{2}$, where $\xi=F^{1 / 4}$ (see Table I).

b Computed in Ref. [16] using the configuration-interaction (CI) for He and semi-empirical dipole polarizabilities [17] for the noble gases, and in the present work, using empirically-adjusted CI + many-body theory data [31] for the alkaline-earth atoms.

${ }^{\text {c }}$ For Ps-H and Ps-Ps: pseudostate calculations [32]. For all other systems, these values were calculated using the London formula $C_{6}=3 \alpha_{A} \alpha_{B} I_{A} I_{B} /\left[2\left(I_{A}+I_{B}\right)\right]$, where $\alpha_{A, B}$ and $I_{A, B}$ are the static dipole polarizabilities and ionization potentials of the atoms [18]; the polarizabilities were taken from Ref. [28] and the ionization potentials were taken from Ref. [33].

present values for $\mathrm{Kr}$ and Xe being more accurate numerically due to better convergence. The results are also in agreement with calculations made using the relativistic random-phase approximation (RRPA, which also accounts for exchange). Larger differences are to be expected for heavier atoms, since relativistic corrections scale as $(Z / 137)^{2}$, where $Z$ is the nuclear charge. However, even for the heaviest noble-gas atom (Xe) the difference is less than $0.5 \%$. This bodes well for using the dynamic polarizabilities to calculate the $C_{6}$ coefficients for Ps-noble-gas pairs. As expected, for the $n s^{2}$ atoms, the agreement with RRPA is poorer for heavier atoms (since the $s$ electrons are affected more strongly by the relativistic corrections). The agreement with the experimental values for these atoms is also poorer in general. This is related to the smaller ionization potentials and larger effect of the non-RPA correlation effects (e.g., twohole-two-particle excitations) in these systems.

The results for $C_{6}$ are displayed in Table II. Our calculations which use RPAE polarizabilities ("Present" in Table II) are in close agreement with the SE calculations of Mitroy and Bromley [16] for the heavier noble gases; the relative differences for Ps-Ar, Ps-Kr, and Ps-Xe are 2\%, $1 \%$, and $0.3 \%$ respectively. For the lighter noble gases the differences are more significant: $4 \%$ and $11 \%$ for $\mathrm{Ps}_{\mathrm{s}} \mathrm{He}$ and Ps-Ne, respectively. This discrepancy can be traced back to the fact that the RPAE polarizabilities for $\mathrm{He}$ and $\mathrm{Ne}$ are lower than the recommended values, by $5 \%$ and $12 \%$, respectively. Correcting the $\mathrm{RPAE} C_{6}$ value 
for He by the corresponding factor $F$ (see Table I) gives $C_{6}=13.45$, which agrees with the accurate value for $\mathrm{He}$ from Ref. [16] to within $0.6 \%$. However, this crude scaling violates the Thomas-Reiche-Kuhn sum rule, which in the limit of large $\omega$ gives

$$
\alpha(i \omega) \simeq \frac{N}{\omega^{2}}
$$

where $N$ is the number of atomic electrons. A better approach is to scale the dipole matrix elements $\langle\mu\|d\| \nu\rangle$ and $\langle\nu\|A(\omega)\| \mu\rangle$ in Eq. (8) by some factor $\xi$ and the orbital energies in the denominators by $1 / \xi^{2}$. In this case the sum rule is preserved, while the calculated static polarizability is scaled by $\xi^{4}$. The appropriate choice of $\xi$ is given by $\xi=F^{1 / 4}$. Carrying out this scaling for He gives $C_{6}=13.41$, which agrees with the value from Ref. [16] to within $0.3 \%$. This is a clear improvement over the simple scaling by a factor of $F$.

As a further test of the effectiveness of this scaling, the $C_{6}$ constants were calculated for pairs of the noblegas and alkaline-earth-metal atoms. These were compared with data from Ref. [31], wherein several relativistic many-body theory $[29,34]$ and semi-empirical [17] methods were employed. For noble-gas pairs, the minimum relative difference was $0.7 \%$ (for Ar-Ar), while the maximum (for Xe-Xe) was $5 \%$ (due to the use of the dynamical polarizability "normalized" to $\alpha(0)=27.16$ a.u. [17] in Ref. [31], which is lower than the recommended experimental value $\alpha(0)=27.815$ a.u. that we use). For alkaline-earth-metal pairs, the comparison was actually better; the relative differences ranged from $0.2 \%$ (for $\mathrm{Sr}$ $\mathrm{Sr}$ ) to $2.1 \%$ (for Be-Be). This is because we use the same recommended static polarizabilities for the alkaline earth atoms as in Ref. [31].

Scaling the $a b$ initio RPAE polarizabilities in this way produces our best prediction of the $C_{6}$ van der Waals coefficients (third column in Table II). We expect that for the noble-gas atoms these values are accurate to within $1 \%$. The relative differences from the CI/SE values of Mitroy and Bromley for Ne, Ar, Kr, and Xe are 1\%, $0.2 \%$, $1.8 \%$, and $2.6 \%$, respectively. We believe that for $\mathrm{Kr}$ and Xe our best prediction values are superior to those of Ref. [16], where the polarizabilities from Ref. [17] were used.

Since the RPAE polarizabilities of the $n s^{2}$ atoms are greater than the recommended values of $\alpha(0)$ by $15-50 \%$, the calculated $a b$ initio $C_{6}$ values significantly overestimate the true van der Waals coefficients. Given the larger discrepancy for $\alpha(0)$, the use of scaling is a cruder procedure for improving the $C_{6}$ constants. In this case we believe that our best predictions are accurate within few per cent for the alkaline earth atoms, and within 5-10\% for $\mathrm{Zn}$ and $\mathrm{Cd}$.

As a further test of the accuracy of our predictions for the alkaline-earth atoms, we used tabulated dynamic dipole polarizabilities from Ref. [31], which were computed using a combination of relativistic methods, including RRPA, CI and many-body perturbation theory, and further adjusted using accurate theoretical and ex-
TABLE III. Van der Waals $C_{6}$ coefficients for Ps-alkali-metal systems (in atomic units).

\begin{tabular}{ccccccc}
\hline \hline Atom & $\mathrm{Li}$ & $\mathrm{Na}$ & $\mathrm{K}$ & $\mathrm{Rb}$ & $\mathrm{Cs}$ & $\mathrm{Fr}$ \\
\hline$C_{6}{ }^{\mathrm{a}}$ & 462.8 & 505.7 & 765.7 & 846.8 & 1014.6 & 937.5 \\
$C_{6}{ }^{\mathrm{b}}$ & 463.9 & 507.2 & 768.3 & 844.8 & - & - \\
\hline \hline
\end{tabular}

${ }^{\text {a }}$ Computed in the present work using empirically-adjusted many-body theory data [31].

b Semi-empirical calculations from Ref. [16].

perimental data. These values are presented in the fourth column of Table II (lower half). In all cases the difference between these values and our best predictions does not exceed 1\%. Ref. [31] also presents accurate dynamic dipole polarizabilities for the alkali-metal atoms, and we used these to compute the $C_{6}$ coefficients for the Psalkali-metal pairs, see Table III. Comparison with semiempirical calculations presented in Ref. [16] shows excellent agreement.

Looking at the last column in Table II, we see that the London formula does a reasonable job for the more rigid noble-gas atoms, but tends to overestimate the $C_{6}$ coefficients significantly for Ps interacting with $n s^{2}$ atoms.

\section{CONCLUSIONS}

Dynamic dipole polarizabilities over a range of imaginary frequencies were computed numerically exactly for $\mathrm{H}$ and Ps, and by using RPAE for the noble-gas atoms and several other closed-shell atoms. The static polarizabilities for the noble gases deviated from relativistic (RRPA) calculations by no more than $0.5 \%$, and for Ar, $\mathrm{Kr}$, and Xe were within few per cent of experimental values. There was greater error for the other closed-shell atoms, but this was expected; the RPAE method is most suitable for the noble gases.

$A b$ initio calculations of the van der Waals $C_{6}$ coefficients for Ps interactions with these atoms were performed. For the heavier noble gases, close agreement is observed with previous calculations [16]. For the lighter noble gases ( $\mathrm{He}$ and $\mathrm{Ne}$ ) the discrepancies are more significant, which motivates a sum-rule preserving scaling of the dynamical polarizabilities to calculate $C_{6}$ values. Though these data are no longer ab initio, they are expected to the be the most accurate values currently available. For the most part, our values of $C_{6}$ for Ps interactions with the $n s^{2}$ atoms differ significantly from the London values. Here our best predicitons are less accurate (few to $10 \%$ ), but as there are no other theoretical calculations of these data, these values will provide a useful benchmark for future calculations.

It is interesting to discuss the implications of the $C_{6}$ values for low-energy Ps-atom scattering. It is clear from Table II that for more polarizable (and more weakly bound) atoms, the $C_{6}$ values are greater, and that Ps will experience a stronger van der Waals attraction to 
these atoms. However, it can be seen from the parameter $x_{0}$, Eq. (4), which determines the scattering length in Eq. (3), that the increase in $C_{6}$ for such atoms is offset by the increase in the parameter $R_{0}$, which is proportional to the atomic radius. In fact, the latter effect makes $x_{0}$ smaller for the more weakly bound atoms. As a result, the more polarizable atoms are not more attractive for Ps, and will likely have larger positive Ps scattering lengths, due to their larger geometric sizes. This also shows that Ps binding to closed-shell neutral atoms does not occur. Note that this is in contrast to many open-shell atoms, e.g., $\mathrm{Na}, \mathrm{Cu}$, or the halogens, which do bind $\mathrm{Ps}$ by accommodating the extra electron in their valence shell $[35,36]$. However, even in the case of Ps bound states with alkali-metal atoms, the binding energy decreases in the sequence PsLi, PsNa, PsK [37], in spite of the greater values of the $C_{6}$ constant. This is primarily the effect of the increasing atomic radius.

It is hoped that the results presented here will be use- ful for studies of Ps-noble-gas-atom scattering and Ps interactions with $n s^{2}$ and alkali-metal atoms. With little extra work, the method can be extended to calculate quadrupole and higher polarizabilities and determine higher-order van der Waals coefficients $C_{8}$ and $C_{10}$.

\section{ACKNOWLEDGMENTS}

The work of A.R.S. has been supported by the Department of Employment and Learning (Northern Ireland).

\section{Appendix A: RPAE equations}

In the equations for the RPAE dipole matrix element $\langle\nu|A(\omega)| \mu\rangle$, we must distinguish between the hole states, i.e., states below the Fermi level $F$, and particle states, i.e., states above the Fermi level $F$. For $\nu>F$ and $\mu \leq F$ we have

$$
\langle\nu|A(\omega)| \mu\rangle=\langle\nu|d| \mu\rangle+\left(\sum_{\nu^{\prime}>F, \mu^{\prime} \leq F}-\sum_{\mu^{\prime}>F, \nu^{\prime} \leq F}\right) \frac{\left\langle\nu \mu^{\prime}|V| \nu^{\prime} \mu\right\rangle-\left\langle\mu^{\prime} \nu|V| \nu^{\prime} \mu\right\rangle}{\omega-\epsilon_{\nu^{\prime}}+\epsilon_{\mu^{\prime}}+i\left(1-2 n_{\nu^{\prime}}\right) \delta}\left\langle\nu^{\prime}|A(\omega)| \mu^{\prime}\right\rangle,
$$

and a formally identical equation for $\nu \leq F$ and $\mu>F$.

In Eq. (A1), $\langle\nu|d| \mu\rangle$ is the Hartree-Fock dipole matrix element; $\left\langle\nu \mu^{\prime}|V| \nu^{\prime} \mu\right\rangle$ is the Coulomb matrix element, which is defined by

$$
\left\langle\nu \mu^{\prime}|V| \nu^{\prime} \mu\right\rangle \equiv \iint \varphi_{\nu}^{*}(\mathbf{r}) \varphi_{\mu^{\prime}}^{*}\left(\mathbf{r}^{\prime}\right) \frac{1}{\left|\mathbf{r}-\mathbf{r}^{\prime}\right|} \varphi_{\nu^{\prime}}\left(\mathbf{r}^{\prime}\right) \varphi_{\mu}(\mathbf{r}) d^{3} \mathbf{r} d^{3} \mathbf{r}^{\prime}
$$

where the $\varphi_{\nu}$ are single-particle wave functions; $\epsilon_{\nu^{\prime}}$ is the energy of state $\nu^{\prime}$; and

$$
n_{\nu^{\prime}}= \begin{cases}0 & \text { for } \quad \nu^{\prime}>F \\ 1 & \text { for } \quad \nu^{\prime} \leq F\end{cases}
$$

By separating the angular and radial parts in the electronic states $\varphi$, and then integrating over the angular variables and summing over the magnetic quantum numbers and spins, one obtains the RPAE equations for the reduced amplitudes $\langle\nu\|A(\omega)\| \mu\rangle$ in the form

$$
\langle\nu\|A(\omega)\| \mu\rangle=\langle\nu\|d\| \mu\rangle+\frac{1}{3}\left(\sum_{\nu^{\prime}>F, \mu^{\prime} \leq F}-\sum_{\mu^{\prime}>F, \nu^{\prime} \leq F}\right) \frac{\left\langle\nu \mu^{\prime}\left\|U_{1}\right\| \nu^{\prime} \mu\right\rangle\left\langle\nu^{\prime}\|A(\omega)\| \mu^{\prime}\right\rangle}{\omega-\epsilon_{\nu^{\prime}}+\epsilon_{\mu^{\prime}}+i\left(1-2 n_{\nu^{\prime}}\right) \delta},
$$

where

$$
\begin{gathered}
\langle\nu\|d\| \mu\rangle=(-1)^{l_{\nu}} \sqrt{\left[l_{\nu}\right]\left[l_{\mu}\right]}\left(\begin{array}{ccc}
l_{\nu} & 1 & l_{\mu} \\
0 & 0 & 0
\end{array}\right) \int_{0}^{R} P_{\nu}(r) r P_{\mu}(r) d r, \\
\left\langle\nu \mu^{\prime}\left\|U_{1}\right\| \nu^{\prime} \mu\right\rangle=2\left\langle\nu \mu^{\prime}\left\|V_{1}\right\| \nu^{\prime} \mu\right\rangle-3 \sum_{l=0}^{\infty}(-1)^{l-1}\left\{\begin{array}{lll}
l_{\nu} & 1 & l_{\mu} \\
l_{\mu^{\prime}} & l & l_{\nu^{\prime}}
\end{array}\right\}\left\langle\nu \mu^{\prime}\left\|V_{l}\right\| \mu \nu^{\prime}\right\rangle,
\end{gathered}
$$

and

$$
\left\langle\nu \mu^{\prime}\left\|V_{l}\right\| \nu^{\prime} \mu\right\rangle=\sqrt{\left[l_{\nu}\right]\left[l_{\mu^{\prime}}\right]\left[l_{\nu^{\prime}}\right]\left[l_{\mu}\right]}\left(\begin{array}{ccc}
l_{\nu} & l & l_{\mu} \\
0 & 0 & 0
\end{array}\right)\left(\begin{array}{ccc}
l_{\mu^{\prime}} & l & l_{\nu^{\prime}} \\
0 & 0 & 0
\end{array}\right) \int_{0}^{R} \int_{0}^{R} P_{\nu}(r) P_{\mu^{\prime}}\left(r^{\prime}\right) \frac{r_{<}^{l}}{r_{>}^{l+1}} P_{\nu^{\prime}}\left(r^{\prime}\right) P_{\mu}(r) d r d r^{\prime}
$$


are the reduced dipole and Coulomb matrix elements, $P_{\nu}(r)$ are radial wave functions, $\left[l_{\nu}\right] \equiv 2 l_{\nu}+1, r_{>}=\max \left(r, r^{\prime}\right)$, $r_{<}=\min \left(r, r^{\prime}\right)$, and $R$ is the box radius in our $B$-spline-basis implementation.

Introducing vectors $x$ and $y$ for $\langle\nu\|A(\omega)\| \mu\rangle$ for $\nu>F$, $\mu \leq F$ and $\nu \leq F, \mu>F$, respectively, we can write equations (A4) in block matrix form as

$$
\left(\begin{array}{l}
x \\
y
\end{array}\right)=\left(\begin{array}{l}
d \\
d
\end{array}\right)+\left(\begin{array}{cc}
U_{1 a} & U_{1 b} \\
U_{1 b} & U_{1 a}
\end{array}\right)\left(\begin{array}{cc}
\chi_{1} & 0 \\
0 & \chi_{2}
\end{array}\right)\left(\begin{array}{l}
x \\
y
\end{array}\right)
$$

where $\chi_{1}$ and $\chi_{2}$ are the diagonal matrices of energy denominators, $d$ is the vector of Hartree-Fock dipole matrix elements, and the matrices $U_{1 a}$ and $U_{1 b}$ represent the two terms in (A6). This linear equation can be solved numerically for the RPAE dipole amplitudes, and then the dynamic dipole polarizability is calculated from Eq. (8).
[1] G. Laricchia and H. R. J. Walters, Rivista del Nuovo Cimento 35, 305 (2012).

[2] A. Kellerbauer, M. Amoretti, A. Belov, G. Bonomi, I. Boscolo, R. Brusa, M. Büchner, V. Byakov, L. Cabaret, C. Canali, C. Carraro, F. Castelli, S. Cialdi, M. de Combarieu, D. Comparat, G. Consolati, N. Djourelov, M. Doser, G. Drobychev, A. Dupasquier, G. Ferrari, P. Forget, L. Formaro, A. Gervasini, M. Giammarchi, S. Gninenko, G. Gribakin, S. Hogan, M. Jacquey, V. Lagomarsino, G. Manuzio, S. Mariazzi, V. Matveev, J. Meier, F. Merkt, P. Nedelec, M. Oberthaler, P. Pari, M. Prevedelli, F. Quasso, A. Rotondi, D. Sillou, S. Stepanov, H. Stroke, G. Testera, G. Tino, G. Trénec, A. Vairo, J. Vigué, H. Walters, U. Warring, S. Zavatarelli, and D. Zvezhinskij, Nucl. Instrum. Methods B 266, 351 (2008).

[3] G. Consolati, R. Ferragut, A. Galarneau, F. Di Renzo, and F. Quasso, Chem. Soc. Rev. 42, 3821 (2013).

[4] D. W. Gidley, H.-G. Peng, and R. S. Vallery, Ann. Rev. Mat. Res. 36, 49 (2006).

[5] K. Shibuya, T. Nakayama, H. Saito, and T. Hyodo, Phys. Rev. A 88, 012511 (2013).

[6] K. Shibuya, Y. Kawamura, and H. Saito, Phys. Rev. A 88, 042517 (2013).

[7] A. P. Mills, Jr., Rad. Phys. Chem. 76, 76 (2007).

[8] D. B. Cassidy and A. P. Mills, Jr., Phys. Rev. Lett. 100, 013401 (2011).

[9] H. S. W. Massey and C. B. O. Mohr, Proc. Phys. Soc. Section A 67, 695 (1954).

[10] J. Mitroy and I. A. Ivanov, Phys. Rev. A 65, 012509 (2001).

[11] J. Mitroy and M. W. J. Bromley, Phys. Rev. A 67, 034502 (2003).

[12] I. I. Fabrikant and G. F. Gribakin, Phys. Rev. A 90, 052717 (2014).

[13] L. D. Landau and E. M. Lifshitz, Quantum Mechanics: Non-Relativistic Theory, 2nd ed. (Pergamon Press, Oxford, 1965).

[14] P. S. Ganas, Phys. Rev. A 5, 1684 (1972).

[15] G. F. Gribakin and V. V. Flambaum, Phys. Rev. A 48, 546 (1993).

[16] J. Mitroy and M. W. J. Bromley, Phys. Rev. A 68, 035201 (2003).

[17] A. Kumar and W. J. Meath, Mol. Phys. 54, 823 (1985).

[18] F. London, Trans. Faraday Soc. 33, 8b (1937).
[19] M. Ya. Amusia, N. A. Cherepkov, and L. V. Chernysheva, Zh. Eksp. Teor. Fiz 60, 160 (1971), [Sov. Phys. JETP, 33, 90 (1971)].

[20] M. Ya. Amusia, Atomic Photoeffect (Plenum, New York, 1990).

[21] F. Visser, P. E. S. Wormer, and P. Stam, J. Chem. Phys. 79, 4973 (1983).

[22] M. Ya. Amusia, N. A. Cherepkov, and S. Shapiro, Zh. Eksp. Teor. Fiz. 63, 889 (1972), [Sov. Phys. JETP, 36, 468 (1973)].

[23] J. Ludlow, Ph.D. thesis, Queen's University Belfast (2003).

[24] V. B. Berestetskii, E. M. Lifshitz, and L. P. Pitaevskii, Quantum Electrodynamics, 2nd ed. (Pergamon Press, Oxford, 1982).

[25] G. F. Gribakin and J. Ludlow, Phys. Rev. A 70, 032720 (2004).

[26] M. Ya. Amusia and N. A. Cherepkov, Case Studies in Atomic Physics 5, 47 (1975).

[27] D. Kolb, W. R. Johnson, and P. Shorer, Phys. Rev. A 26, 19 (1982).

[28] P. Schwerdtfeger, "Atomic static dipole polarizabilities," in Computational Aspects of Electric Polarizability Calculations: Atoms, Molecules and Clusters, edited by G. Maroulis (IOS Press, Amsterdam, 2006) pp. 1-32, updated static dipole polarizabilities are available as a PDF file from the CTCP website at Massey University: http://ctcp.massey.ac.nz/dipole-polarizabilities.

[29] S. G. Porsev and A. Derevianko, Zh. Eksp. Teor. Fiz. 129, 227 (2006), [Sov. Phys. JETP 102, 195 (2006)].

[30] R. P. McEachran, A. D. Stauffer, and S. Greita, J. Phys. B 12, 3119 (1979).

[31] A. Derevianko, S. G. Porsev, and J. F. Babb, At. Data Nucl. Data Tables 96, 323 (2010).

[32] D. W. Martin and P. A. Fraser, J. Phys. B 13, 3383 (1980).

[33] D. R. Lide, ed., CRC Handbook of Chemistry and Physics, 89th ed. (CRC Press, Boca Raton, FL, 20082009).

[34] S. G. Porsev and A. Derevianko, Phys. Rev. A 65, 020701 (2002).

[35] J. Mitroy, M. W. J. Bromley, and G. G. Ryzhikh, J. Phys. B 35, R81 (2002).

[36] J. A. Ludlow and G. F. Gribakin, Int. Rev. At. Mol. Phys. 1, 73 (2010), see also arXiv:physics/1002.3125v1.

[37] J. Mitroy and G. Ryzhikh, J. Phys. B 32, 3839 (1999). 